\title{
IMAGEN CORPORAL Y MODELOS ESTÉTICOS CORPORALES EN LA ADOLESCENCIA Y LA JUVENTUD
}

\section{Igor Esnaola Etxanlz}

Universidad del Pais Vasco

\section{RESUMEN}

En esta investigación se analiza la relación entre la imagen corporal (EDI-2) y los modelos estéticos corporales (CIMEC-26). Por un lado, se analiza las diferencias en función del género, y por otro, las diferencias entre dos grupos de edad, adolescentes y jóvenes. La muestra está compuesta por 627 adolescentes y 271 jóvenes. La media de edad de los adolescentes es de 15.33 años y la de los jóvenes 22.49 años. Los resultados indican que las mujeres, tanto en la adolescencia como en la juventud, muestran mayor insatisfaccion corporal y mayor influencia de los modelos estéticos corporales. En cuanto a la edad, existen diferencias significativas en el EDI-2. Los varones adolescentes se perciben con mayor absesión por la delgadez y sentimientos de ineficacia que los varones jóvenes; las mujeres adolescentes se perciben con mayor obsesión por la delgadez, insatisfacción corporal y sentimientos de ineficacia. En cuanto al CIMEC26 , solo se han encontrado diferencias significativas en la muestra de los varones. Los varones jóvenes están más afectados por la influencia de los modelos sociales y la influencia de las situaciones sociales que los adolescentes varones.

Palabras clave: IMAGEN CORPORAL, MODELOS ESTÉTICOS CORPORALES, ADOLESCENCIA, JUVENTUD. 


\section{SUMMARY}

The relationship between the body image (EDI-2) and the sociocultural influences of aesthetic models (CIMEC-26) are analyzed. On the one hand, the differences of gender, and on the other hand, the differences between two age of groups, adolescents and youth. The sample consists of 627 adolescent and 271 young people. The average age of adolescents is 15.33 years old and in young poople 22.49 years old. The results show that the females, in adolescence and youth, have more body dissatisfaction and are more influenced by socio-cultural aesthetic models. Regarding aged, significant differences were found in EDI-2. Adolescent males have more drive for thinness and ineffectiveness than youth males, and adolescent females have more drive for thinness, body dissatisfaction and ineffectiveness than youth females. In CIMEC26, the only significant differences have been found in males. Youth males are affected more than adolescent males in influence of social models and influence of social situations.

Key words: BODY IMAGE, AESTHETIC BODY MODELS, ADOLESCENCE, YOUTH.

\section{INTRODUCCIÓN}

El estudio de los distintos aspectos de la imagen corporal ha adquirido creciente interés a raíz del incremento de problemas de salud asociados con ella, como son los trastornos de la alimentación, la adicción al ejercicio físico o ejercicio físico excesivo, vigorexia, dismorfofobia, abuso de dietas y esteroides, etc. (Cruz y Maganto, 2002; Raich, Torras y Figueras, 1996). Cuando la preocupación por poseer un cuerpo ideal no disminuye con el paso de los años sino que se incrementa, puede convertirse en un gran impedimento para el óptimo desarrollo de la persona (Cruz y Maganto, 2002). La teoria de la discrepancia entre el yo real y el yo ideal dice que la presión sociocultural hacia un modelo corporal delgado potencia la preocupación por el peso. Esto conduce a un buen número de mujeres 
a compararse con el ideal socialmente aceptado. La probable discrepancia entre el cuerpo real y el cuerpo ideal genera insatisfacción corporal. El tener que ajustarse a ciertos valores e ideales de belleza homogeneizados en el entorno social lleva a un estado de evaluación corporal continuo que tiene un efecto tremendamente negativo, generando una gran angustia, ansiedad y tristeza (Ballester y Guirado, 2003).

Un problema actual, propio de la sociedad de consumo, es el culto al cuerpo y a su valor estético. La obsesión por el cuerpo perfecto ha conducido, especialmente a grupos de jóvenes, a una tiranía de la belleza. Ello dificulta en unos casos, e impide en otros, la coexistencia de otros valores de mayor urgencia y necesidad en la formación humana. Tener un físico agradable se ha convertido en una "obligación", pues ello supone prestigio, seguridad y superioridad, aunque a veces, se alcance a costa del sacrificio de otros valores más humanizantes. Nunca el cuerpo ha influido tanto en el yo, y en el autoconcepto. La mujer hoy se siente obligada a vivir en la perfección. Hoy el cuerpo, "la percha", en mayor o menor grado, se encuentra asociado a todo o casi todo. La ética ha cedido su valor, en buena parte, a la estética. Hoy, para muchos, la búsqueda de la perfección moral ha sido sustituida por la perfección corporal (Gervilla, 2002).

Las consecuencias de esta presión social se observa en los resultados de diferentes estudios donde se ha encontrado que el $82 \%$ de los varones y el $93 \%$ de las mujeres manifiestan y experimentan una intensa orientación hacia la apariencia física, y en otras investigaciones se constata que el $95 \%$ de las mujeres sobrestiman el volumen de sus cuerpos (Moreno, 1999). Estos resultados son preocupantes; es normal que una persona obesa esté preocupada por su figura, pero lo realmente preocupante es el gran porcentaje de población con peso normal, que muestra las mismas actitudes respecto al cuerpo que una persona obesa o incluso peores, y están siguiendo dietas aún más restrictivas (Moreno, 1999).

Los medios de comunicación social son el cauce fundamental de este conocimiento $y$, en buena parte, la causa del problema. La publicidad lo invade todo, y no podemos escapar totalmente a su influencia. Toro, Cervera y Pérez (1988) hicieron un análisis de la publicidad "pro esbeltez" incluida en las diez revistas "femeninas" con 
más de 100.000 ejemplares editados que se publican en España, y observaron que uno de cada cuatro anuncios invitaba directa o indirectamente a perder peso. Las manifestaciones artísticas de la estética corporal son constantes en el cine, en el mundo de la canción y de la moda, en los presentadores/as de TV, en los anuncios de productos light, cosméticos, dietas, cirugía estética, etc. Vivimos en una época tan sensual que sólo los cuerpos guapos son válidos para vender coches, detergentes, viviendas, productos alimentarios, etc. Una imagen, hoy más que nunca, vende más que mil palabras (Gervilla, 2002).

Entre las hipótesis explicativas de la insatisfacción de las mujeres se encuentra el impacto que tiene el modelo estético corporal vigente en la cultura occidental (Raich, 2000). En nuestra cultura, actualmente uno de los factores más influyentes puede ser la presión cultural hacia la esbeltez y la estigmatización de la obesidad, lo que Vandereycken y Meerman (1984) llamaron la "cultura de la delgadez". Sin embargo, hay que tener en cuenta que estudios de comparación transcultural muestran que la presión pro esbeltez existe en determinadas sociedades, pero no en otras (Nasser, 1988).

Las teorías socioculturales defienden que el ideal estético corporal propuesto por la sociedad, y vehiculizado por los medios de comunicación, es interiorizado por la mayoría de las mujeres de una cultura debido a la elevada dependencia existente entre autoestima y atractivo físico en las mujeres (Raich, 2000). El ideal estético corporal imperante en la actualidad cae dentro del peso muy bajo: "ser bella es igual a ser delgada" (Raich, 2000). La "cultura de la delgadez" (Vandereycken y Meerman, 1984) precoriza para la mujer cuerpos delgados, y con formas poco redondeadas (Cruz y Maganto, 2002). A los chicos, a pesar de que la presión social no es tan importante, también la sociedad les exige tener un determinado físico. En los hombres los cánones de belleza siguen primando el "vigor varonil" que subyace a la fuerza corporal, fundamentalmente focalizada en un torso musculoso y atlético (Raich, 2000). Como es sabido, en las mujeres el esquema corporal, a pesar de ser más nítido, se percibe fragmentado, a diferencia de los varones que tienden a una percepción holística e integrada (Cruz y Maganto, 2002). El modelo estético masculino es mucho más saludable que el femenino (Gervilla, 2002). 
No es extraño que las mujeres estén insatisfechas con su peso, ya que el modelo propuesto por los medios de comunicación es mucho más delgado que el normal; y cuando el propio cuerpo no es acorde con los modelos vigentes en la sociedad, los avances de la ciencia se ponen a su servicio, al servicio del cuerpo perfecto. Hoy la ciencia consigue el cuerpo que queremos, a gusto del consumidor; ya el ser humano no contempla su físico como algo definitivo e inalterable (Gervilla, 2002).

Los medios de comunicación ejercen una intensa presión social al transmitir el mensaje, fundamentalmente dirigido a las mujeres, de que un físico delgado proporciona felicidad, ya que éste, por lo general, no sólo se asocia con belleza y atractivo sexual, lo que facilita ser amada, sino que también implica tener más poder y autocontrol, concretamente, un pleno control sobre el cuerpo que informa de una subjetividad perfecta y poderosa; la imagen corporal no está sólo ligada a la belleza física, sino a características de personalidad altamente valoradas socialmente (Martínez, 2001). La belleza del cuerpo, en buena parte, condiciona, y hasta determina, el placer, el éxito, las amistades, el sexo, etc. Hoy quienes gozan de "buena presencia" poseen, y sólo por ello, mayores posibilidades laborales, de relaciones humano-afectivas y de aceptación social. De este modo, subordinan a este "dios" la comida, el descanso, el vestido, el placer, el dinero, etc. Todo, o casi todo, al servicio de la fuerza de su belleza (Gervilla, 2002). Cuanto más piensa la mujer que lo que es gordo es feo, lo delgado bello y lo bello es bueno, más va a intentar adelgazar y va a sentirse más preocupada por poco que engorde (Raich, 2000).

Algunos estudios han demostrado la marcada preferencia pro esbeltez que se ha desarrollado en las últimas décadas, a diferencia de lo que pasa en los países que tienen dificultades en la obtención de comida. Garner, Garfinkel, Swarts y Thompson (1980) llevaron a cabo un estudio en el que revisaron el peso y la altura de las modelos de Playboy y de las concursantes y ganadoras del concurso de Miss América desde 1959 hasta 1978, y no sólo encontraron una disminución de peso de 3,4 kg., sino también de las medidas de pecho y caderas. Wiseman, Gray, Mosiman y Ahrens (1992) han replicado este estudio y han deducido que los pesos de las misses no sólo han disminuido (entre 1979 y 1988), sino que están entre un 13 y un 19 por 100 por 
debajo del peso normativo. Gordon (1994), también afirma que hace una generación, las modelos pesaban por término medio, un $8 \%$ menos que las mujeres normales, mientras hoy pesan un $23 \%$ menos. Ballester y Guirado (2003) afirman que en los niños de edades tempranas (11 años) ya se aprecian conductas alimentarias problemáticas que podrian reflejar una cierta gestación de trastornos más graves.

Asimismo, va creciendo de manera alarmante una consideración hacia la obesidad o simplemente el sobrepeso que tiende a estigmatizarla cada vez más (Raich, 2000). En el V Congreso Estatal de Asociaciones contra la Anorexia y Bulimia (San Sebastián, 2002) Joseph Toro afirmó que la preocupación por el peso y la figura se encuentra en preadolescentes de 9 años de edad, que sitúan su peso ideal por debajo de la media. Ser obeso/a o simplemente presentar sobrepeso ( $y$ aún en nuestra cultura se consideran gruesas personas con un peso absolutamente dentro de los límites de la normalidad) es sinónimo de ser una persona "dejada" o descuidada, poco saludable y evidentemente fea y poco atractiva (Raich, 2000). Es sinónimo de ser "dejada" ya que es extraordinariamente fácil perder peso (sólo con pasearte por delante de las farmacias de nuestro pais o de algunas tiendas de alimentos puede verse que tomando no sé qué comida light o consumiendo determinado tipo de alimentos se pueden perder muchos kilos). Está en el ambiente que uno pesa lo que quiere pesar, puesto que es posible manipular el propio cuerpo y darle la forma adecuada. También puede cambiarse gracias a la cirugía estética y haciendo un intenso y saludable ejercicio físico. Es ésta una de las falacias que más se ha transmitido en nuestra cultura, la posible manipulación de nuestro cuerpo. Parece que una de las empresas más rentables son las que se dedican a reducir o repartir el peso. El negocio está cifrado en billones de pesetas e incluye todas las variedades de industrias de productos de consumo bajos en calorías, centros de estética, centros de gimnasia y otros. Estudios controlados demuestran que un grandísimo porcentaje de nuestro peso corporal, así como su distribución, lo debemos a nuestra herencia genética . Del mismo modo que heredamos el color de ojos y de cabellos y piel, la altura y otras características, también tendemos a heredar el peso medio. Ante su manipulación o cambio (cosa que todas las revistas 
proclaman que es tan fácilmente alcanzable), tiende a mantenerse por mecanismos ancestrales, heredados directamente de nuestros abuelos, que pasaban grandes temporadas de hambruna.

Ser obeso es también sinónimo de poca salud (Raich, 2000). Ser grueso o presentar sobrepeso se ha convertido en el principal objetivo de intervención por parte de muchos médicos y personal sanitario. Si bien es cierto que una gran obesidad favorece enfermedades y algunas de ellas graves, lo contrario no es cierto: cuanto más delgado más saludable. Un cierto nivel de sobrepeso correlaciona con longevidad (Raich, 2000). También se ha visto que las personas muy delgadas suelen morir antes que las que no lo son tanto. Finalmente, las personas obesas son, por definición, feas. Vivimos en una sociedad delgadofílica, que parece olvida cualquier otra característica estética frente a la delgadez para definir a la belleza. Por lo menos a la belleza femenina. Además, el deseo de delgadez actual ya no supone en la mayoría de las personas el anhelo por destacar y ser valorado de modo particular, sino el ser suficientemente aceptado, 0 incluso el no sentirse rechazado (Moreno, 1999).

Como se ha comentado anteriormente, el físico de las mujeres que aparecen en los medios de comunicación en general y en la publicidad en particular, es cada vez más irreal lo cual implica riesgos para la salud de las mujeres al buscar un ideal corporal inalcanzable, y además supone una importante dedicación temporal al cuidado del cuerpo y la búsqueda de la belleza (Santiso, 2001). El cuidado del físico es una nueva obligación más para las mujeres que la autora Naomi Wolf (1991) ha llamado "el tercer turno laboral".

Tales hechos ponen de manifiesto una de las paradojas más graves e inmorales de nuestro tiempo, pues mientras gran parte de la humanidad muere de hambre, o bien se nutre de modo insuficiente por falta de alimentos, otro sector del mundo, gracias a su opulencia, pasa también hambre para adelgazar y así adecuar su imagen a los modelos de belleza vigentes (Gervilla, 2002).

Aprender a construir modelos de mujeres alternativos a los que nos ofrece la sociedad de consumo y más acordes con los de la vida cotidiana es un reto que tenemos por delante. Como dice Rita Freedman (1991) "tenemos el derecho y la responsabilidad de compararnos con modelos reales. Es vital, para ello, entender que no 
es necesario cambiar nuestros cuerpos sino las reglas de este absurdo juego (Santiso, 2001).

Antecedentes de la investigación. Se ha sugerido que las presiones sociales a favor de la delgadez son particularmente influyentes durante la adolescencia y la juventud, al ser uno de los períodos críticos en el desarrollo de la identidad y del rol sexual. Esta imagen negativa se convierte en un problema que afecta en mayor grado a las mujeres que a los hombres.

Hecho que se ha relacionado con una diferente presión y valoración sociocultural de los estereotipos masculinos y femeninos considerados como normativos. La influencia de los medios de comunicación también se ha demostrado diferente en ambos sexos. Se ha constatado, por ejemplo, que existe una correlación positiva entre el peso real y el sobrepeso percibido con el tiempo dedicado a mirar los anuncios de televisión en mujeres adolescentes pero no en hombres (de Gracia, Marc6, Fernández y Juan, 1999).

Estos autores (1999) analizaron las relaciones entre el autoconcepto físico, la imagen corporal y los modelos estéticos corporales. Los resultados indicaron que en el grupo de chicas se observó una fuerte correlación entre malestar por la imagen corporal, la insatisfacción por el propio cuerpo y una mayor receptividad a los mensajes publicitarios y verbales relacionados con la delgadez. El grupo de adolescentes que manifiestan una mayor presión del modelo estético, presentaban también una menor valoración del autoconcepto físico en casi todas sus dimensiones, una mayor insatisfacción con la propia imagen corporal y un índice de masa corporal significativamente más bajo.

Myers y Biocca (1992) afirman que ver 30 minutos de televisión y publicidad puede alterar la percepción del cuerpo de las mujeres. Martínez, Toro y Salamero (1996) analizaron la correlación entre el EAT (Eating Attitudes Test) y el CIMEC (Cuestionario de Influencias sobre el Modelo Estético Corporal), encontrando correlaciones muy significativas. Afirman que cuanto mayor es la presencia e interiorización de influencias culturales favorecedoras de la estética de la delgadez tanto más relevante es la sintomatología alimentaria. Sin embargo, también cabe que las cosas sucedan de otro modo; la paciente anoréxica, una vez instaurado el trastorno, a causa del mismo bien pudiera estar mucho más sensibilizada y receptiva a este género de factores ambientales, 
facilitándose así una mayor interiorización de los mismos. No obstante, lo más probable es que se den ambos fenómenos a la vez.

Martínez, Toro, Salamero, Blecua y Zaragoza (1993) afirman que la influencia de la publicidad está significativamente más extendido en el grupo de entre 18 y 24 años que en el de adolescentes. Cualquier información verbal que exista, parece tener mucha mayor difusión entre las jóvenes que entre las adolescentes. Prácticamente dos tercios de la muestra admiten su gusto por los cuerpos femerinos que, exhibidos públicamente, son extremadamente delgados.

Vázquez, Ocampo, López, Mancilla y Leticia (2002) analizaron la influencia de los modelos estéticos en la insatisfacción corporal en estudiantes de danza entre los 10-18 años, afirmando mayor insatisfacción corporal a medida que hay una mayor influencia de los modelos estéticos de "delgadez".

Tiggemann (2003) investigó la relación entre la exposición a los medios de comunicación y el descontento con el cuerpo y los trastornos alimentarios. Mientras que tanto las variables de exposición a los medios correlacionaron con el descontento con el cuerpo, el modelo de correlación era muy diferente con las otras variables. En concreto, la cantidad de revistas leídas, pero no la televisión que se visualizaba, estuvo correlacionada positivamente con la internalización de ideales de delgadez. Por otra parte, el tiempo que se pasaba frente a la televisión estuvo correlacionado negativamente con conciencia de ideales socioculturales y de autoestima. Tiggemann (2003) concluye que los procesos por los cuales la televisión y las revistas impactan de modo diferente en el descontento corporal son diferentes. La relación entre la exposición a las revistas y la insatisfacción corporal es mediada por la internalización de ideales de delgadez, la cual no es el caso de la televisión.

\section{Objetivos}

El objetivo de la investigación que se presenta es analizar la influencia de dos variables en la relación de la imagen corporal y los modelos estéticos corporales. Por un lado, el género; y por otro lado, la variable edad. Para ello se han seleccionado dos grupos de edad: la adolescencia y la juventud. 


\section{MÉTODO}

\section{Participantes}

La muestra de esta investigación está compuesta por 627 adolescentes $(69.8 \%)$ y 271 jóvenes $(30.2 \%)$. En función del género, la muestra se distribuye de esta manera: mujeres adolescentes 327 (36.4\%), varones adolescentes $300(33.4 \%)$, mujeres jóvenes 151 (16.8\%) y jóvenes varones $120(13.4 \%)$. El rango de edad de los adolescentes es de 12 a 18 años, con una media de edad de 15.33 años; el rango de los jóvenes se sitúa entre los 19 y 28 años, con una media de edad de 22.49 años.

\section{Instrumentos}

En esta investigación se administraron dos cuestionarios:

- Eating Disorder Inventory (EDI-2; Garner, 1998): Inventario compuesto de 91elementos distribuidos en 11 escalas diferentes relacionadas con los trastornos de la conducta alimentaria. En esta investigación se seleccionaron cuatro subescalas: obsesión por la delgadez, insatisfacción corporal, bulimia y sentimientos de ineficacia.

- Cuestionario de Influencias sobre el Modelo Estético Corporal (CIMEC; Toro, Salamero y Martinez, 1995): Elaborado y evaluado en una población española, consta de 40 preguntas que evalúan la ansiedad por la imagen corporal, así como la influencia de los modelos estéticos corporales y las situaciones sociales sobre el modelo de delgadez. Los autores señalan como una alternativa para el análisis 26 preguntas agrupadas en cinco factores: malestar por la imagen corporal, influencia de la publicidad, influencia de los mensajes verbales, influencia de los modelos sociales e influencia de las situaciones sociales. En esta investigación se aplicó esta versión reducida. Presenta una aceptable consistencia interna tanto en población clínica como en población normal. En la versión utilizada en este estudio se modificó la redacción de aquellos items que en su origen sólo podían ser contestados por mujeres formulándolos todos de tal forma que resultasen adecuados tanto para hombres como para mujeres. La consistencia interna medida 
por el alpha de Cronbach obtuvo resultados satisfactorios de .82 para los varones y .90 para las mujeres.

\section{Procedimiento}

En primer lugar se contactó con los centros y se informó de la finalidad del estudio. Después de obtener la autorización de los centros, se concertó la cita. Los cuestionarios fueron repartidos en las aulas por el autor de este estudio, después de dar una explicación sobre su contenido y aclarar dudas. Durante el proceso colectivo, se aclararon dudas que surgieron al rellenar los cuestionarios y se revisaron si estaban cumplimentados adecuadamente a la hora de entregarlos.

Se realizó la prueba de Kolmogorov-Smimov para ver si se cumplían las condiciones paramétricas. Los resultados indicaron que ninguna subescala analizada cumplia las condiciones paramétricas. Por tanto, a la hora de analizar las diferencias entre los grupos se realizó la prueba Mann-Whitney.

\section{RESULTADOS}

En primer lugar, se exponen los resultados del análisis en función del género en ambos grupos de edad.

En la adolescencia, existen diferencias significativas entre varones y mujeres en las subescalas obsesión por la delgadez $z$ (-8.435) ( $p$ $=.001)$, insatisfacción corporal z $(-8.734)(p=.001)$, malestar por la imagen corporal z (-8.697) ( $p=.001)$, influencia de la publicidad $z$ ($8.140)(p=.001)$, influencia de los mensajes verbales $z(-10.057)(p$ $=.001)$ e influencia de los modelos sociales z $(-3.314)(p=.001)$. En todos los casos, las mujeres puntúan más que los varones. Esto significa que, en la adolescencia, las mujeres se perciben con mayor insatisfacción corporal, mayor malestar por la imagen corporal, les influye más la publicidad, los mensajes verbales y los modelos sociales que a los varones.

En la juventud, existen diferencias significativas en función del género en absesión por la delgadez $z(-4.882)(p=.001)$, insatisfacción corporal $z(-3.482)(p=.001)$, malestar por la imagen corporal $z(-5.332)$ ( $p$ $=.001)$, influencia de la publicidad $z(-4.659)(p=.001)$ e influencia de 
TABLA 1. Puntuaciones en función del género en cada grupo de edad.

\begin{tabular}{|c|c|c|c|c|c|c|}
\hline & & & \multicolumn{2}{|c|}{ Adolescencia } & \multicolumn{2}{|c|}{ Juventud } \\
\hline Test & Subescala & & Mujeres & Varones & Mujeres & Varones \\
\hline \multirow{12}{*}{ EDI-2 } & \multirow{3}{*}{$\begin{array}{l}\text { Obsesión por } \\
\text { la delgadez }\end{array}$} & $\begin{array}{c}\mathbf{M} \\
(\mathbf{D} . \mathrm{T})\end{array}$ & $\begin{array}{c}4.53 \\
(4.850)\end{array}$ & $\begin{array}{c}1.51 \\
(2.226)\end{array}$ & $\begin{array}{c}2.99 \\
(4.078)\end{array}$ & $\begin{array}{c}.97 \\
(2.054)\end{array}$ \\
\hline & & $\mathbf{z}$ & \multicolumn{2}{|c|}{-8.435} & \multicolumn{2}{|c|}{-4.882} \\
\hline & & Sig. & \multicolumn{2}{|c|}{$.001 * * *$} & \multicolumn{2}{|c|}{$.001 * * *$} \\
\hline & \multirow[t]{3}{*}{ Bulimia } & $\begin{array}{c}\mathbf{M} \\
\text { (D.T) }\end{array}$ & $\begin{array}{c}1.22 \\
(2.127) \\
\end{array}$ & $\begin{array}{c}1.33 \\
(2.227) \\
\end{array}$ & $\begin{array}{c}1.49 \\
(2.983)\end{array}$ & $\begin{array}{c}.87 \\
(1.328)\end{array}$ \\
\hline & & $x$ & \multicolumn{2}{|c|}{-.178} & \multicolumn{2}{|c|}{-1.328} \\
\hline & & Sig. & \multicolumn{2}{|c|}{.858} & \multicolumn{2}{|c|}{.184} \\
\hline & \multirow{3}{*}{$\begin{array}{l}\text { Insatisfacción } \\
\text { corporal }\end{array}$} & $\begin{array}{c}\mathbf{M} \\
(\mathrm{D}, \mathrm{T})\end{array}$ & $\begin{array}{c}8.50 \\
(7.261)\end{array}$ & $\begin{array}{c}3.84 \\
(5.172)\end{array}$ & $\begin{array}{c}6.99 \\
(6.992)\end{array}$ & $\begin{array}{c}3.74 \\
(4.766)\end{array}$ \\
\hline & & $\mathbf{z}$ & \multicolumn{2}{|c|}{-8.734} & \multicolumn{2}{|c|}{-3.482} \\
\hline & & Sig. & \multicolumn{2}{|c|}{$.001 * *$} & \multicolumn{2}{|c|}{$.001^{* * *}$} \\
\hline & \multirow{3}{*}{$\begin{array}{l}\text { Sentimientos } \\
\text { de ineficacia }\end{array}$} & $\begin{array}{c}\mathbf{M} \\
(\mathrm{D} . \mathrm{T})\end{array}$ & $\begin{array}{c}3.06 \\
(3.960)\end{array}$ & $\begin{array}{c}2.61 \\
(3.557)\end{array}$ & $\begin{array}{c}2.10 \\
(3.180)\end{array}$ & $\begin{array}{c}2.11 \\
(4.806)\end{array}$ \\
\hline & & $\mathbf{z}$ & \multicolumn{2}{|c|}{-1.651} & \multicolumn{2}{|c|}{-1.822} \\
\hline & & Sig. & \multicolumn{2}{|c|}{.099} & \multicolumn{2}{|c|}{.069} \\
\hline \multirow{15}{*}{ CIMEC } & \multirow{3}{*}{$\begin{array}{l}\text { Malestar por } \\
\text { la imagen } \\
\text { corporal }\end{array}$} & $\begin{array}{c}\mathbf{M} \\
(\mathrm{D} . \mathrm{T})\end{array}$ & $\begin{array}{c}5.87 \\
(3.945)\end{array}$ & $\begin{array}{c}2.93 \\
(2.698)\end{array}$ & $\begin{array}{c}6.02 \\
(3.700)\end{array}$ & $\begin{array}{c}3.53 \\
(2.990)\end{array}$ \\
\hline & & $\mathbf{z}$ & \multicolumn{2}{|c|}{-8.697} & \multicolumn{2}{|c|}{-5.332} \\
\hline & & Sig. & \multicolumn{2}{|c|}{$.001 * * *$} & \multicolumn{2}{|c|}{$.001^{* * *}$} \\
\hline & \multirow{3}{*}{$\begin{array}{l}\text { Influencia de } \\
\text { la publicidad }\end{array}$} & $\begin{array}{c}\mathrm{M} \\
\text { (D.T) }\end{array}$ & $\begin{array}{c}3.16 \\
(3.396)\end{array}$ & $\begin{array}{c}1.22 \\
(2.306) \\
\end{array}$ & $\begin{array}{c}2.62 \\
(3.050)\end{array}$ & $\begin{array}{c}.88 \\
(1.285) \\
\end{array}$ \\
\hline & & $\mathbf{z}$ & \multicolumn{2}{|c|}{-8.140} & & \\
\hline & & Sig. & .00 & $* *$ & & \\
\hline & $\begin{array}{l}\text { Influencia de } \\
\text { los mensajes }\end{array}$ & $\begin{array}{c}\mathrm{M} \\
(\mathrm{D} . \mathrm{T})\end{array}$ & $\begin{array}{c}1.93 \\
(1.697)\end{array}$ & $\begin{array}{c}.62 \\
(1.082)\end{array}$ & $\begin{array}{c}1.74 \\
(1.599)\end{array}$ & $\begin{array}{c}1.12 \\
(1.416)\end{array}$ \\
\hline & verbales & $\mathbf{z}$ & & 57 & & \\
\hline & & Sig. & .00 & $* *$ & & \\
\hline & $\begin{array}{l}\text { Influencia de } \\
\text { los modelos }\end{array}$ & $\begin{array}{c}\text { M } \\
\text { (D.T) }\end{array}$ & $\begin{array}{c}3 . \overline{79} \\
(1.597)\end{array}$ & $\begin{array}{c}3 . \overline{28} \\
(1.725)\end{array}$ & $\begin{array}{c}3.77 \\
(1.466)\end{array}$ & $\begin{array}{c}3.70 \\
(1.324)\end{array}$ \\
\hline & sociales & $\mathbf{x}$ & & & & \\
\hline & & Sig. & .00 & $* *$ & & \\
\hline & $\begin{array}{c}\text { Influencia de } \\
\text { las }\end{array}$ & $\begin{array}{c}\text { M } \\
\text { (D.T) }\end{array}$ & $\begin{array}{c}1.57 \\
(1.339)\end{array}$ & $\begin{array}{c}1.32 \\
(1.061)\end{array}$ & $\begin{array}{c}1.45 \\
(1.154)\end{array}$ & $\begin{array}{c}1.41 \\
(1.018)\end{array}$ \\
\hline & situaciones & $x$ & & & & \\
\hline & sociales & Sig. & & & & \\
\hline
\end{tabular}

${ }^{*} p<.05{ }^{* *} p<.01{ }^{* * *} p<.001$

los mensajes verbales $z(-3.157)(p=.002)$. Como ocurría en la adolescencia, las mujeres jóvenes se perciben con mayor insatisfacción corporal, mayor malestar por la imagen corporal, les influye más la publicidad y los mensajes verbales que a los varones. 
TABLA 2. Puntuaciones en la adolescencia y juventud agrupados por género.

\begin{tabular}{|c|c|c|c|c|c|c|}
\hline & & & \multicolumn{2}{|c|}{ Varones } & \multicolumn{2}{|c|}{ Mujeres } \\
\hline Test & Subescala & & Adolescencia & Juventud & Adolescencin & Juventud \\
\hline \multirow{12}{*}{ EDI-2 } & \multirow{3}{*}{$\begin{array}{l}\text { Obsesión } \\
\text { por la } \\
\text { delgadez }\end{array}$} & $\begin{array}{c}\mathbf{M} \\
(\mathbf{D} . \mathrm{T})\end{array}$ & $\begin{array}{c}1.51 \\
(2.226)\end{array}$ & $\begin{array}{c}.97 \\
(2.054)\end{array}$ & $\begin{array}{c}4.53 \\
(4.850)\end{array}$ & $\begin{array}{c}2.99 \\
(4.078)\end{array}$ \\
\hline & & $\mathbf{z}$ & \multicolumn{2}{|c|}{-2.665} & \multicolumn{2}{|c|}{-3.342} \\
\hline & & Sig. & \multicolumn{2}{|c|}{$.008^{* *}$} & \multicolumn{2}{|c|}{$.001^{* * *}$} \\
\hline & \multirow[t]{3}{*}{ Bulimia } & $\begin{array}{c}\mathbf{M} \\
(\mathbf{D} \cdot \mathbf{T})\end{array}$ & $\begin{array}{c}1.33 \\
(2.227) \\
\end{array}$ & $\begin{array}{c}.87 \\
(1.328) \\
\end{array}$ & $\begin{array}{c}1.22 \\
(2.127) \\
\end{array}$ & $\begin{array}{c}1.49 \\
(2.983) \\
\end{array}$ \\
\hline & & $\mathrm{z}$ & \multicolumn{2}{|c|}{-.446} & \multicolumn{2}{|c|}{-1.185} \\
\hline & & Sig. & \multicolumn{2}{|c|}{.656} & \multicolumn{2}{|c|}{.236} \\
\hline & \multirow{3}{*}{$\begin{array}{l}\text { Insatisfacei } \\
\text { on corporal }\end{array}$} & $\begin{array}{c}\mathrm{M} \\
(\mathbf{D} . \mathrm{T})\end{array}$ & $\begin{array}{c}3.84 \\
(5.172) \\
\end{array}$ & $\begin{array}{c}3.74 \\
(4.766) \\
\end{array}$ & $\begin{array}{c}8.50 \\
(7.261) \\
\end{array}$ & $\begin{array}{c}6.99 \\
(6.992) \\
\end{array}$ \\
\hline & & $\mathbf{z}$ & \multicolumn{2}{|c|}{-.324} & \multicolumn{2}{|c|}{-2.251} \\
\hline & & Sig. & \multicolumn{2}{|c|}{.746} & \multicolumn{2}{|c|}{$.024^{*}$} \\
\hline & \multirow{3}{*}{$\begin{array}{l}\text { Sentimiento } \\
\text { s de } \\
\text { ineficacia }\end{array}$} & $\begin{array}{c}\mathbf{M} \\
(\mathbf{D}, \mathbf{T})\end{array}$ & $\begin{array}{c}2.61 \\
(3.557)\end{array}$ & $\begin{array}{c}2.11 \\
(4.806)\end{array}$ & $\begin{array}{c}3.06 \\
(3.960)\end{array}$ & $\begin{array}{c}2.10 \\
(3.180)\end{array}$ \\
\hline & & $\mathbf{z}$ & \multicolumn{2}{|c|}{-3.169} & \multicolumn{2}{|c|}{-2.789} \\
\hline & & Sig. & \multicolumn{2}{|c|}{$.002^{* *}$} & \multicolumn{2}{|c|}{$.005^{* *}$} \\
\hline \multirow{15}{*}{ CIMEC } & \multirow{3}{*}{$\begin{array}{c}\text { Malestar } \\
\text { por la } \\
\text { imngen } \\
\text { corporal }\end{array}$} & $\begin{array}{c}\mathbf{M} \\
(\mathbf{D} . \mathbf{T})\end{array}$ & $\begin{array}{c}2.93 \\
(2.698)\end{array}$ & $\begin{array}{c}3.53 \\
(2.990)\end{array}$ & $\begin{array}{c}5.87 \\
(3.945)\end{array}$ & $\begin{array}{c}6.02 \\
(3.700)\end{array}$ \\
\hline & & $\mathbf{z}$ & \multicolumn{2}{|c|}{-1.739} & \multicolumn{2}{|c|}{-.456} \\
\hline & & Sig. & \multicolumn{2}{|c|}{.082} & \multicolumn{2}{|c|}{.649} \\
\hline & \multirow{3}{*}{$\begin{array}{l}\text { Influencia } \\
\text { de la } \\
\text { publicidad }\end{array}$} & $\begin{array}{c}\text { M } \\
\text { (D.T) }\end{array}$ & $\begin{array}{c}1.22 \\
(2.306)\end{array}$ & $\begin{array}{c}.88 \\
(1.285)\end{array}$ & $\begin{array}{c}3.16 \\
(3.396)\end{array}$ & $\begin{array}{c}2.62 \\
(3.050)\end{array}$ \\
\hline & & $\mathbf{z}$ & \multicolumn{2}{|c|}{-.057} & -1 & \\
\hline & & Sig. & .9 & & .1 & \\
\hline & $\begin{array}{c}\text { Influencia } \\
\text { de los }\end{array}$ & $\begin{array}{c}\mathrm{M} \\
\text { (D. I) }\end{array}$ & $\begin{array}{c}.62 \\
(1.082)\end{array}$ & $\begin{array}{c}1.12 \\
(1.416)\end{array}$ & $\begin{array}{c}1.93 \\
(1.697)\end{array}$ & $\begin{array}{c}1.74 \\
(1.599)\end{array}$ \\
\hline & mensajes & $\mathbf{z}$ & -3.5 & & -1. & \\
\hline & verbales & Sig. & .001 & & .3 & \\
\hline & $\begin{array}{c}\text { Infuencia } \\
\text { de los }\end{array}$ & $\begin{array}{c}\mathbf{M} \\
(\mathbf{D} \cdot \mathbf{T})\end{array}$ & $\begin{array}{c}3.28 \\
(1.725)\end{array}$ & $\begin{array}{c}3.70 \\
(1.324) \\
\end{array}$ & $\begin{array}{c}3.79 \\
(1.597)\end{array}$ & $\begin{array}{c}3.77 \\
(1.466)\end{array}$ \\
\hline & modelos & $\mathbf{z}$ & -2.2 & & -.3 & \\
\hline & socisles & Sig. & .02 & & .7 & \\
\hline & $\begin{array}{l}\text { Influencia } \\
\text { de las }\end{array}$ & $\begin{array}{c}\mathbf{M} \\
(\mathbf{D} . \mathbf{T})\end{array}$ & $\begin{array}{c}1.32 \\
(1.061)\end{array}$ & $\begin{array}{c}1.41 \\
(1.018)\end{array}$ & $\begin{array}{c}1.57 \\
(1.339)\end{array}$ & $\begin{array}{c}1.45 \\
(1.154)\end{array}$ \\
\hline & situaciones & $\mathbf{z}$ & -.7 & & -.5 & \\
\hline & sociales & Sig. & .4 & & .6 & \\
\hline
\end{tabular}

" $p<.05$ "* $p<.01$ "**p $<.001$

Después de analizar las diferencias en función del género, se analizaron las diferencias entre los dos grupos de edad, adolescencia y juventud. Teniendo en cuenta que en los análisis anteriores se encontraron diferencias significativas en función del género, en estos 
análisis se decidió analizar los resultados de forma independiente para cada género, y no comparar los grupos adolescente y joven globalmente. Por tanto, los resultados se exponen de forma independiente para cada género. Los resultados se exponen en la tabla 2.

Entre los varones, se han encontrado diferencias significativas en obsesión por la delgadez z (-2.665) $(p=.008)$, sentimientos de ineficacia $z(-3.169)(p=.002)$, influencia de los mensajes verbales $z(-3.553)(p$ $=.001)$, e influencia de los modelos sociales z $(-2.231)(p=.026)$. Los adolescentes varones se perciben con mayor obsesión por la delgadez y sentimientos de ineficacia que los varones jóvenes. Sin embargo, los varones jóvenes están más afectados por la influencia de los mensajes verbales y los modelos sociales. Entre las mujeres, se han encontrado diferencias significativas en obsesión por la delgadez $z(-3.342)(p=.001)$, insatisfacción corporal $z(-2.251)(p=.024)$ y sentimientos de ineficacia $z(-2.789)(p=.005)$. Las mujeres adolescentes se perciben con mayor obsesión por la delgadez, insatisfacción corporal y sentimientos de ineficacia que las jóvenes. En el caso de las mujeres no se han encontrado diferencias significativas en las subescalas del Cuestionario de Influencias sobre el Modelo Estético Corporal (CIMEC). Finalmente, se exponen las correlaciones de Pearson entre las subescalas del Eating Disorder Inventory (EDI-2) y el Cuestionario de Influencias sobre el Modelo Estético Corporal (CIMEC). Los resultados pueden observarse en la tabla 3.

TABLA 3. Correlaciones entre el EDI-2 y el CIMEC

\begin{tabular}{|c|c|c|c|c|c|}
\hline & & $\begin{array}{c}\text { Obsesión por } \\
\text { la delgadez }\end{array}$ & Bulimin & $\begin{array}{c}\text { Insatisfacción } \\
\text { corporal }\end{array}$ & $\begin{array}{l}\text { Sentimientos } \\
\text { de ineficacia }\end{array}$ \\
\hline \multirow{2}{*}{$\begin{array}{l}\text { Malestar par la } \\
\text { imagen corparal }\end{array}$} & Correlación & \multirow{2}{*}{$\begin{array}{c}.697^{* * *} \\
.001\end{array}$} & \multirow{2}{*}{$\begin{array}{c}.274^{* * *} \\
.001\end{array}$} & \multirow{2}{*}{$\begin{array}{c}.671^{*} * * \\
.001\end{array}$} & \multirow{2}{*}{$\begin{array}{c}.334 * * * \\
.001\end{array}$} \\
\hline & Sig. & & & & \\
\hline \multirow{2}{*}{$\begin{array}{c}\text { Influencia de la } \\
\text { publicidad }\end{array}$} & Correlación & \multirow{2}{*}{$\begin{array}{c}.634^{* * *} \\
.001\end{array}$} & \multirow{2}{*}{$\begin{array}{c}.357^{* * * *} \\
.001\end{array}$} & \multirow{2}{*}{$\begin{array}{c}.482 * * * \\
.001\end{array}$} & \multirow{2}{*}{$\begin{array}{c}.292 * * * \\
.001\end{array}$} \\
\hline & Sig. & & & & \\
\hline \multirow{2}{*}{$\begin{array}{l}\text { Influencia de los } \\
\text { mensajes verbales }\end{array}$} & Correlación & \multirow{2}{*}{$\begin{array}{c}.499^{* * *} \\
.001\end{array}$} & \multirow{2}{*}{$\begin{array}{c}.214^{* * *} \\
.001\end{array}$} & \multirow{2}{*}{$\begin{array}{c}.359^{* * *} \\
.001\end{array}$} & \multirow{2}{*}{$\begin{array}{c}.179 * * * \\
.001\end{array}$} \\
\hline & Sig. & & & & \\
\hline \multirow{2}{*}{$\begin{array}{l}\text { Influencia de los } \\
\text { modelos sociales }\end{array}$} & Correlación & \multirow{2}{*}{$\begin{array}{c}.248 * * * \\
.001\end{array}$} & \multirow{2}{*}{$\begin{array}{c}.210^{* * *} \\
.001\end{array}$} & \multirow{2}{*}{$\begin{array}{c}.095^{* 4} \\
.009\end{array}$} & \multirow{2}{*}{$\begin{array}{c}.099^{* 4} \\
.007\end{array}$} \\
\hline & Sig. & & & & \\
\hline \multirow{2}{*}{$\begin{array}{c}\text { Influencia de las } \\
\text { situaciones } \\
\text { sociales }\end{array}$} & Correlación & \multirow{2}{*}{$\begin{array}{c}.405^{* *} \\
.001\end{array}$} & \multirow{2}{*}{$\begin{array}{c}.183^{* 4 *} \\
.001\end{array}$} & \multirow{2}{*}{$\begin{array}{c}.309^{* * *} \\
.001\end{array}$} & \multirow{2}{*}{$\begin{array}{c}.257^{* * *} \\
.001\end{array}$} \\
\hline & Sig & & & & \\
\hline
\end{tabular}

"p $<.05 * " p<.01 * * p<.001$ 
Todas las subescalas del Eating Disorder Inventory (EDI-2) correlacionan significativamente con todas las subescalas del Cuestionario de Influencias sobre el Modelo Estético Corporal (CIMEC). Los resultados indican una importante asociación entre una alta obsesión por la delgadez, insatisfacción corporal y sentimientos de ineficacia, con altas puntuaciones en la irifluencia de la publicidad, los mensajes verbales, los modelos sociales y las situaciones sociales.

\section{DISCUSIÓN}

Nuestros resultados indican diferencias significativas en función de la primera variable analizada, el género, a favor de los varones tanto en la adolescencia como en la juventud. En el grupo adolescente se encontraron diferencias significativas en 6 de las 9 subescalas analizadas y en la juventud en 5 de las 9 subescalas analizadas (véase tabla 1). Las mujeres se perciben con mayor insatisfacción corporal y están más influenciadas por los modelos estéticos corporales. Por tanto, la variable género es un factor significativo en la relación entre la imagen corporal y los modelos estéticos corporales.

Los resultados de esta investigación confirman los encontrados en la literatura, en las que se afirma que las mujeres están más influenciadas por los modelos estéticos corporales y están más insatisfechas con su imagen corporal que los varones (de Gracia et al., 1999).

En cuanto a la variable edad, entre los varones se encontraron diferencias significativas en 4 de las 9 subescalas y entre las mujeres en 3 de las 9 subescalas (véase tabla 2). Hay que señalar que en el Eating Disorder Inventory (EDI-2) los jóvenes (tanto varones como mujeres) puntúan mejor que los adolescentes. Los adolescentes se perciben con mayor obsesión por la delgadez y sentimientos de ineficacia que los jóvenes (en ambos sexos), y las adolescentes mujeres se perciben con mayor insatisfacción corporal que las mujeres jóvenes. Sin embargo, en el Cuestionario de Influencias sobre el Modelo Estético Corporal (CIMEC), únicamente se encontraron diferencias significativas entre los varones. Los jóvenes están más afectados por los mensajes verbales y los modelos sociales que los adolescentes, en la misma dirección que apuntaban Martínez, Toro y Salamero (1993). 
Por tanto, se puede concluir, que la variable edad es un factor significativo en cuanto a la imagen corporal, ya que los jóvenes de ambos sexos se perciben mejor que los adolescentes; sin embargo, en cuanto a la influencia de los modelos estéticos corporales, la variable edad no es un factor significativo en la muestra de las mujeres.

En los varones, los jóvenes varones están más afectados que los adolescentes varones por los mensajes verbales y los modelos sociales.

En el análisis correlacional, los resultados han sido los esperados, ya que las subescalas del Eating Disorder Inventory (EDI-2) correlacionan significativamente con las del Cuestionario de Influencias sobre el Modelo Estético Corporal (CIMEC). Esto significa que los sujetos que se perciben con mayor insatisfacción corporal, son aquellos a los que los modelos estéticos corporales y la influencia de la publicidad más les afecta.

Estos resultados nos deberían ayudar, en primer lugar a los profesionales de la psicología en la elaboración de programas de prevención, sobre todo para las mujeres que son las que están más afectadas. Todo ello, con el objetivo de que las mujeres aprendan a compararse con modelos reales y comprendan que es necesario cambiar la situación actual. Lo saludable psicológicamente sería ofrecer criterios y valores de referencia sobre las que asentar la autoestima y el autoconcepto, más acordes con las cualidades psicológicas y humanas de la persona, y más alejados de una imagen física sobre la que no se tiene muchas posibilidades de maniobra y de manipulación, y que en definitiva, refleja sólo la parte más exterior y superficial de una persona.

\section{REFERENCIAS BIBLIOGRÁFICAS}

Ballester, R. y Guirado, M. C. (2003). Detección de conductas alimentarias de riesgo en niños de once a catorce años. Psicothema, 15 (4), 556-562.

Corral, S., González, M., Pereña, J. y Seisdedos, N. (1998). EDI-2. Inventario de trastomos de la conducta alimentaria. Manual. Madrid: TEA.

Cruz, S. y Maganto, C. (2002). Índice de masa corporal, preocupación por la delgadez y satisfacción corporal en adolescentes. Revista de Psicologra General y Aplicada, 55 (3), 455-473. 
De Gracia, M., Marcó, M., Fernández, M. J. y Juan, J. (1999). Autoconcepto físico, modelo estético e imagen corporal en una muestra de adolescentes. Psiquis, 20 (1), 15-26.

Freedman, R. (1991). Amar nuestro cuerpo. Guia práctica para mujeres. Cómo gustarnos a nosotras mismas. Barcelona: Paidós Ibérica.

Garner, D. M., Garfinkel, P. E., Schwartz, D. y Thompson, M. (1980). Cultural expectations of thinness in women. Psychological Reports, $47,483-491$.

Gervilla, E. (2002). La tiranía de la belleza, un problema educativo hoy. La estética del cuerpo como valor y como problema. Teoría de la Educación, 14, 185-206.

Gordon, R. A. (1994). Anorexia y bulimla. Anatomía de una epidemia social. Barcelona: Editorial Ariel.

Martínez Benlloch, I. (2001). Género, desarrollo psicosocial y trastornos de la imagen corporal. Madrid: Instituto de la Mujer.

Martínez, E., Toro, J. y Salamero (1996). Influencias socioculturales favorecedoras del adelgazamiento y sintomatología alimentaria. Revista de Psiquiatría de la Facultad de Medicina de Barcelona, 23 (5), 125-133.

Martínez, E., Toro, J., Salamero, M., Blecua, M. J. y Zaragoza, M. (1993). Influencias socioculturales sobre las actitudes y conductas femeninas relacionadas con el cuerpo y la alimentación. Revista de Psiquiatría de la Facultad de Medicina de Barcelona, 20 (2), 5165.

Moreno, M. E. (1999). El impacto de los factores socioculturales en los trastornos alimentarios. Informació Psicológica, 70, 50-54.

Myers, P. y Biocca, F. A. (1992). The elastic body image: the effects of television advertising and programming on body image distorsions in young women. Journal of Communication, 42, 899-905.

Nasser, M. (1988). Eating disorders: the cultural dimension. Social Psychiatry and Psychiatric Epidemiology, 23 (3), 184-187.

Raich, R. M. (2000). Imagen corporal. Conocer y valorar el propio cuerpo. Madrid: Ediciones Pirámide.

Raich, R. M., Torras, J. y Figueras, M. (1996). Estudio de la imagen corporal y su relación con el deporte en una muestra de estudiantes universitarios. Análisis y Modificación de Conducta, 22 (85), 603-626. 
Santiso, R. (2001). Las mujeres en la publicidad: análisis, legislación y aportaciones para un cambio. Acciones e Investigaciones Sociales, $13,43-60$.

Tiggemann, M. (2003). Media exposure, body dissastisfaction and disordered eating: televisión and magazines are not the same. European Eating Disorders Review, 11 (5), 418-430.

Toro, J., Cervera, M. y Pérez, P. (1988). Body shape, publicity and anorexia nervosa. Social Psychiatric Epidemiology, 23, 132-136.

Toro, J., Salamero, M. y Martínez, E. (1995). Evaluación de las influencias socioculturales sobre el modelo estético corporal en la anorexia nerviosa. Revista de Psiquiatría de la Facultad de Medicina de Barcelona, 22, 205-214.

Vandereycken, W. y Meerman, R. (1984). Anorexia nervosa: Is prevention possible? Intemational Joumal of Psychiatry in Medicine, 3, 15-24.

Vázquez, R., Ocampo, M. T., López, X., Mancilla, J. M. y Leticia, G. (2002). La influencia de modelos estéticos e insatisfacción corporal en estudiantes de danza con trastorno alimentario. Psicología Conductual, 10 (2), 355-369.

Vélez, Y. M. y Herrasti, E. (2002). La influencia de los medios de comunicación en la imagen corporal. Interpsiquis.

Ventura, L. (2000). La tiranía de la belleza. Las mujeres ante los modelos estéticos. Barcelona: Plaza y Janés.

Wiseman, C. V., Gray, J. J., Mosimann, J. E. y Ahrens, A. H. (1992). Cultural expectations of thinness in women: an update. Intemational Journal of Eating Disorders, 11, 85-89.

Wolf, N. (1991). El mito de la belleza. Barcelona: Emecé editores. 